\title{
Self-assembly of ellipsoidal particles at fluid-fluid interfaces with an empirical pair potential
}

Article

Accepted Version

Creative Commons: Attribution-Noncommercial-No Derivative Works 4.0

Luo, A., Vermant, J., Ilg, P., Zhang, Z. and Sagis, L. M. C. (2019) Self-assembly of ellipsoidal particles at fluid-fluid interfaces with an empirical pair potential. Journal of Colloid and Interface Science, 534. pp. 205-214. ISSN 0021-9797 doi: https://doi.org/10.1016/j.jcis.2018.08.114 Available at https://centaur.reading.ac.uk/78871/

It is advisable to refer to the publisher's version if you intend to cite from the work. See Guidance on citing.

To link to this article DOI: http://dx.doi.org/10.1016/j.jcis.2018.08.114

Publisher: Elsevier

All outputs in CentAUR are protected by Intellectual Property Rights law, including copyright law. Copyright and IPR is retained by the creators or other copyright holders. Terms and conditions for use of this material are defined in the End User Agreement.

www.reading.ac.uk/centaur 
Central Archive at the University of Reading

Reading's research outputs online 


\title{
Self-assembly of ellipsoidal particles at fluid-fluid interfaces with an empirical pair potential
}

\author{
Alan M. Luo, Jan Vermant \\ ETH Zürich, Department of Materials, CH-8093 Zürich, Switzerland. \\ Patrick Ilg \\ School of Mathematical and Physical Sciences, University of Reading, Reading, RG6 6AX, \\ United Kingdom. \\ Zhenkun Zhang \\ Institute of Polymer Chemistry, College of Chemistry, Nankai University, Tianjin 300071, \\ China. \\ Leonard M.C. Sagis \\ Physics and Physical Chemistry of Foods, Wageningen University, Bornse Weilanden 9, \\ 6708 WG Wageningen, The Netherlands.
}

\begin{abstract}
Colloidal particles adsorbed at fluid-fluid interfaces interact via mechanisms that can be specific to the presence of interfaces, for instance, lateral capillary interactions induced by nonspherical particles. Capillary interactions are highly relevant for self-assembly and the formation of surface microstructures, however, these are very challenging to model due to the multibody nature of capillary interactions. This work pursues a direct comparison between our computational modelling approach and experimental results on surface microstructures formed by ellipsoidal particles. We begin by investigating the accuracy of using pairwise interactions to describe the multibody capillary interaction by contrasting exact two- and three-particle interaction energies and we find that the pairwise approximation appears reasonable for the experimentally relevant configurations studied. We then develop an empirical pair potential and use it in MonteCarlo type simulations to efficiently model the stucture formation process for relevant particle properties such as aspect ratio, contact angle and surface cover-
\end{abstract}


age, and succeed in reproducing our experimental observations where we spread sterically-stabilised ellipsoidal particles onto an oil-air interface at high surface coverage. At lower surface coverages, we find that the self-assembly process falls into the diffusion-limited colloid aggregation universality class.

Keywords: elsarticle.cls, LATEX, Elsevier, template

2010 MSC: 00-01, 99-00

\section{Introduction}

Control and understanding of how particles at a fluid-fluid interface interact and self-assemble is a key topic in both fundamental and applied soft matter research. 1] In particular, adsorbed particles can stabilise foams and emulsions

5 against phase separation [2, 3, 4, 5, therefore it must be of no surprise that they are of crucial interest for numerous industries.

The particle-stabilising effect is generally attributed to a kinetic barrier (pictured as an 'armored' bubble or droplet) formed by the adsorbed particles, thereby preventing coalescence of droplets [6], although for very specific systems, a thermodynamically stable Pickering emulsion has been achieved [7. However, it now seems that a sufficient condition for droplet stabilisation is a yield stress interface, which can even be achieved with sub-monolayer coverage [8, 9, 10. Here the surface structure must play an important role in determining the mechanical behaviour of the interface.

Colloidal forces can be strongly modified when particles are present at the interface between two different fluids, as is well known for the electrostatic forces [11, 12, 13, 14, but there are also colloidal interactions which are specific to the nature of the interface. Lateral capillary interactions arise because of deformations of the interface by the presence of particles [15, 16] and physical origins of these deformations include flotation forces, particle shape, surface chemistry, surface roughness and electric charge. Flotation forces are relevant for larger particles, which can reduce their gravitational potential energy by coming together, leading to an effective attractive force - this is also the origin 
of the so-called 'cheerios effect', where floating objects (like the aforementioned breakfast cereal) are observed to aggregate [17. This effect is, however, expected to be negligible for colloidal-sized particles [18, where wetting behaviour plays a more important role. The effect of wetting can be boiled down to a contact angle $\theta$, determined by the Young-Dupré equation

$$
\gamma_{1}-\gamma_{2}-\gamma \cos \theta=0
$$

15 energy between particle and fluid 2, and $\gamma$ is the surface tension between the two fluids. Since the interface must contact the colloid at the contact angle, its profile is deformed. This effect was used to help explain the shape of aggregates formed by mosquito eggs at the air-water interface [19] and more recently the lead to an undulating contact line, which distorts the meniscus 21] and has been used as a simple and robust way to impart emulsion stability [22, 23]. We note that the interface can also be deformed by the presence of charges on the particles if one of the bulk phases contains electrolytes, such as for 25 a water-air or water-oil system [6, 24, 25, 26]. Here the physical mechanism underlying the interface deformation is related to the gathering of counterions on the electrolyte side to screen the particle charge, which results in a pressure field acting on the particle and interface, causing the interface to become deformed (an electrodipping force). The screening effect leads to a leading order dipoledipole electrostatic repulsion at large separations [12, 27, 28].

Since structure formation is a direct result of particle-particle interactions, there is great interest in establishing interaction potentials between two adsorbed particles. The capillary interaction has been inferred experimentally by observing the motion of particles as they come together by Loudet et al. [29] 35 and measured directly using optical tweezers by Park et al. [30. The theory of capillary interactions between two particles is well developed and consists of either solving an energy minimisation problem where the energy of the interface and particles is considered and generally consists of solving the Young-Laplace 
equation with non-trivial boundary conditions on the particles due to the contact angle [15, 24, 31, or by a 'force approach' based on mechanical equilibrium of the system 32 .

However, analytic results for the meniscus deformation due to specific particle geometries are only generally available following simplifications (small gradients, small aspect ratio) 33] and in asymptotic limits (large separation distance)

45 [21, 34. The interaction energy between particles was calculated numerically for various particle geometries and contact angles in Refs. [35, 36, 37. Meniscus deformations of a single particle due to gravity, electric charge, wettability and shape, as well as interactions between particles, have all been studied both experimentally and theoretically [15, 21, 29, 24, 38, 33, 32. The theory behind capillary interactions is well understood, with the main difficulty being in how to efficiently model large-scale structure formation.

We are interested in the effect of particle geometry (aspect ratio) and wetting behaviour (contact angle) on capillary interactions, which are expected to be an important factor in structure formation on fluid interfaces. We consider hard prolate ellipsoids with contact angle $\theta$. This leads to the interface becoming deformed, as it must satisfy the contact angle boundary conditions on the particles while minimising its free energy. Interestingly, the interaction between ellipsoidal particle varies from attractive to repulsive depending on their relative orientation and is quadrupolar to leading order 33

$$
U_{\text {quad }}=-3 \pi \gamma\left(\Delta u_{\max }\right)^{2}\left(\frac{r_{i j}}{R}\right)^{-4} \cos \left(2 \phi_{i}+2 \phi_{j}\right),
$$

where $\Delta u_{\max }$ is the maximum difference in mensicus height on the particle, $r_{i j}$ is the center-to-center separation distance, $R$ is the particle radius and $\phi_{i}, \phi_{j}$ are the orientations of particles $i$ and $j$ respectively.

Given the severe unreliability of the quadrupole approximation for small separations and the prohibitive computational cost of solving the Young-Laplace equation numerically for many particles in order to simulate the structure formation process, we prefer a different approach. Our approach consists of developing an empirical pair potential for ellipsoidal particles, fitting it to exact numeri- 
cal calculations of ellipsoid pairs at different separation distances and relative orientations, which goes beyond the rather inaccurate but typically assumed quadrupolar pair potential 33] by taking into account the ellipsoidal geometry. Our pair potential is then used in sophisticated Virtual Move Monte-Carlo (VMMC) simulations to simulate structure and aggregate formation with an 65 approximation of realistic dynamics [39, 40, 41, which we expect to more accurately treat capillary interactions than the Lattice-Boltzmann simulations of particle-stabilised emulsions of Harting et al. [42, 43, 44].

The validity of using pair potentials to describe multibody capillary interactions depends on the validity of the superposition or Nicolson approximation 45], where the interface profile is assumed to be the sum of interface profiles originating from individual particles. This is expected to be accurate for small deformations of the interface and has been used extensively in theoretical efforts to describe the interaction between two adsorbed particles, for instance, in Refs. [21, 33, 34, 46. For floating sub-millimeter spherical particles, the force between two particles calculated using the superposition approximation is accurate to within a few percent compared to the exact solution [47, although for when the interface deformation is due to electrocapillarity, the validity depends on whether the whole system is in mechanical isolation 48.

Since nearly all previous work is focused on two particle interactions (with so the notable exceptions of Ref. 34, which found some multibody effects for 3 and 4 spherical particles and Ref. [19, which looked more at the aggregation of several ellipsoids), we investigate the use of pair potentials to describe multibody capillary interactions by considering exact numerical results for two and three particles in various configurations in order to validate the use of pair potentials in our VMMC simulations. Our results for the three-particle interaction energies build upon the results in Ref. [19] and help to establish the energetically favoured configurations.

This paper is organised in the following manner: Section 2 deals with the validity of using pair potentials to describe the formally multibody capillary interaction, Section 3 introduces our empirical pair potential, we present results 
concerning self-assembly and structure formation in Section 4 followed by a comparison with experimental results.

\section{Validity of the pairwise approximation}

The capillary interaction arises due to the interaction between meniscus deformations of multiple particles. The free energy $\Delta F$ of a system of $N$ particles adsorbed on a fluid-fluid interface consists of the sum of the surface energy between two phases 1 and 2 and the wetting energies between the phases and the particles

$$
\Delta F=\gamma \Delta A_{\text {men }}+\sum_{i=1}^{N}\left(\gamma_{1} \Delta A_{1, i}+\gamma_{2} \Delta A_{2, i}\right),
$$

where $\Delta A_{\text {men }}$ denotes the change in meniscus surface area, $\Delta A_{1, i}$ and $\Delta A_{2, i}$ denote the change in contact area between the particle $i$ and phase 1 and 2 respectively, and we have assumed $N$ identical particles on the interface. How this free energy behaves when the particle positions and orientations are varied gives rise to effective multibody interparticle interactions. Note that the pairwise approximation is equivalent to the superposition approximation in that the contact line on each particle is unaffected by the meniscus distortion of the other particles. This approximation is violated at very close separations but we minimise this effect by using an empirical pair potential fitted to highly accurate numerical data.

We consider how accurately pair interaction potentials reflect the exact three-particle interaction energy for various three-particle configurations and a range of parameters (contact angle $\theta$, aspect ratio $k$ ). The prolate ellipsoid is defined by a semi-major axis length $a$ and a semi-minor axis length $b$ so that the aspect ratio $k=a / b>1$.

\subsection{Methodology - Surface Evolver}

To obtain the exact two- and three-particle interaction energies numerically, we use the Surface Evolver (version 2.70a) [49]. The meniscus is first discretised 
into Lagrange elements while the contact angle boundary condition on the solid particles is modelled by a constraint energy integral along the contact line that accounts for the wetting energy, namely the contact angle and wetted area. The particle's positions and orientations are fixed. The software uses gradient descent and nonlinear Hessian steps to optimise the position of the contact line and the mensicus profile in order to minimise the free energy Eq. (3). We use adaptive meshing, with a finer mesh closer to the particles. We begin the minimisation procedure on a mesh comprised of linear Lagrange elements, and finish it on a fine mesh of second order Lagrange elements, allowing very precise determination of the meniscus profile and total energy of the system. We impose a far-field (at a radius of $70 a$ away from the center of mass of the particles) flat interface boundary condition, although we do allow the height of the interface at the boundary to vary. Note that the particles are fixed in a given orientation and we do not study tilting out of the interface. This is equivalent to allowing the particle height to vary and find its optimum position with respect to the interface. Reference energies were determined by placing the particles far apart, typically $r_{i j} \approx 40 a$. Our two-particle interaction energies are in accordance with the results reported by Dasgupta et al. [37] and Botto et al. [36].

\subsection{Results and discussion}

We begin by accurately determining the interaction potentials for a variety of two-particle configurations. We then simulate a selection of three-particle configurations and compare the exact three-particle interaction energies with the sum of two-particle interaction energies. The two-particle and three-particle configurations are shown in Fig. 1. In addition, the side-side-side (SSS) and tip-tip-tip (TTT) configurations were simulated for different interparticle separation distances. These configurations were chosen due to their relevance in experimental work, for example see Fig. 8 in the review article by Botto et 


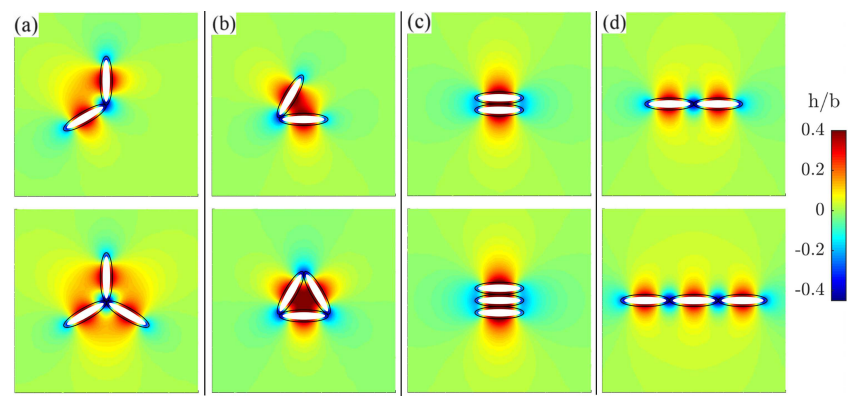

Figure 1: The meniscus deformation due to ellipsoidal particles with $k=4, \theta=40^{\circ}$ in various configurations. Black outlines show the touching particles, white shows the removed surface area of the fluid interface. Two- and three-particle configurations (a) flower, (b) triangle, (c) SSS and (d) TTT.

al. $[35]^{1}$

We characterise the error in assuming pairwise interactions by a percentage relative error, defined as $100 \times\left(E_{3}-\sum_{i}^{3} \sum_{j=i+1}^{3} E_{i j}\right) / E_{3}$ where $E_{3}$ is the threeparticle multibody interaction energy and $E_{i j}$ is the pairwise interaction energy between particles $i$ and $j$. Each of these energies is determined by numerical simulations to a very high level of precision. The SSS and TTT configurations are characterised by two distances $d_{1}$ and $d_{2}$, where $d_{1}$ denotes the distance between the furthermost particles and $d_{2}$ is the distance between the first and middle particle.

The exact three-particle interaction energies are compared with the sum of pairwise interactions for the SSS and TTT configurations in Fig. 2a and for the flower and triangle configurations in Fig. 33 . The trends in the three-particle interaction energies are clearly reproduced by the sum of pairwise interactions and, in terms of the absolute values, the two do not differ significantly. The relative error for the four different three-particle configurations are presented

\footnotetext{
${ }^{1}$ In addition to these stable and metastable configurations, we also studied a selection of unstable three-particle configurations, namely STS, TST, SST and TTS. Those results are not presented here but the approximation of using pairwise energies to describe the full multibody interaction energy also appears reasonable.
} 


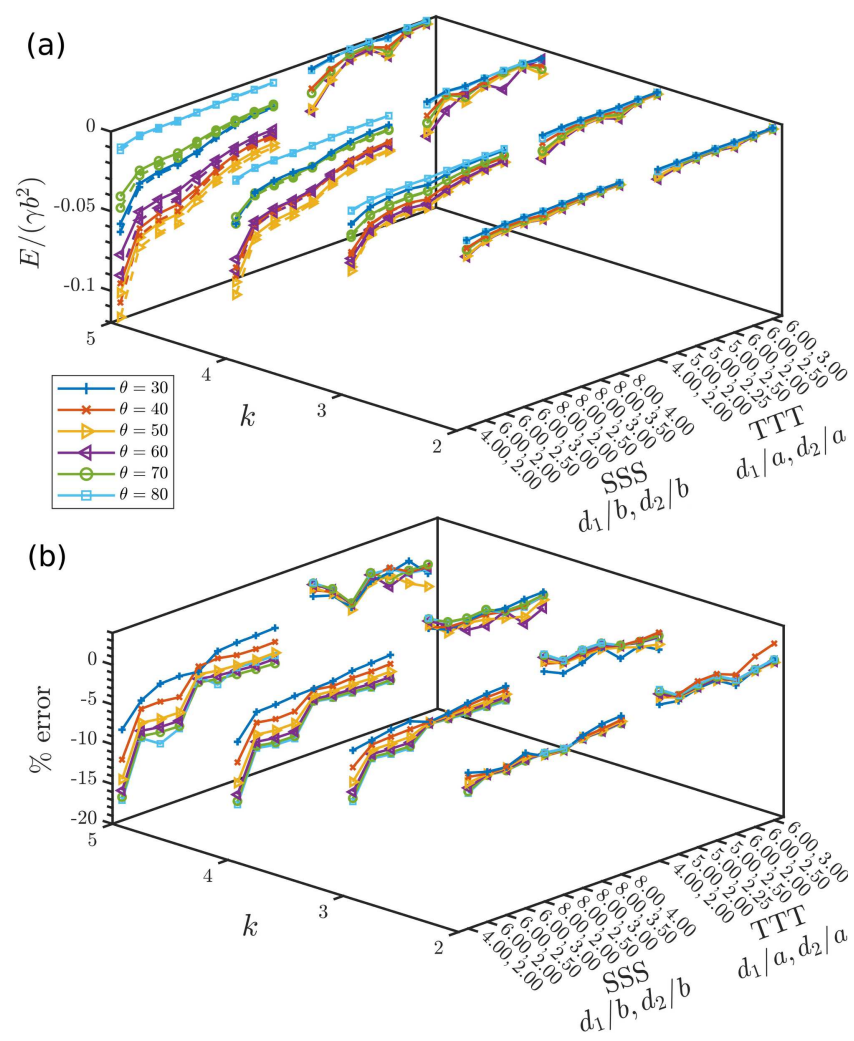

Figure 2: (a) Comparison between the exact three-particle interaction energies (solid lines) and the sum of pairwise interaction energies (dashed lines) for various aspect ratios $k$, contact angles $\theta$ and separation distances $d_{1}, d_{2}$ for particles in the SSS and TTT configurations. (b) Percentage relative error (see main text) of the pairwise approximation for the same $k, \theta, d_{1}$, $d_{2}$ as in the upper panel. 

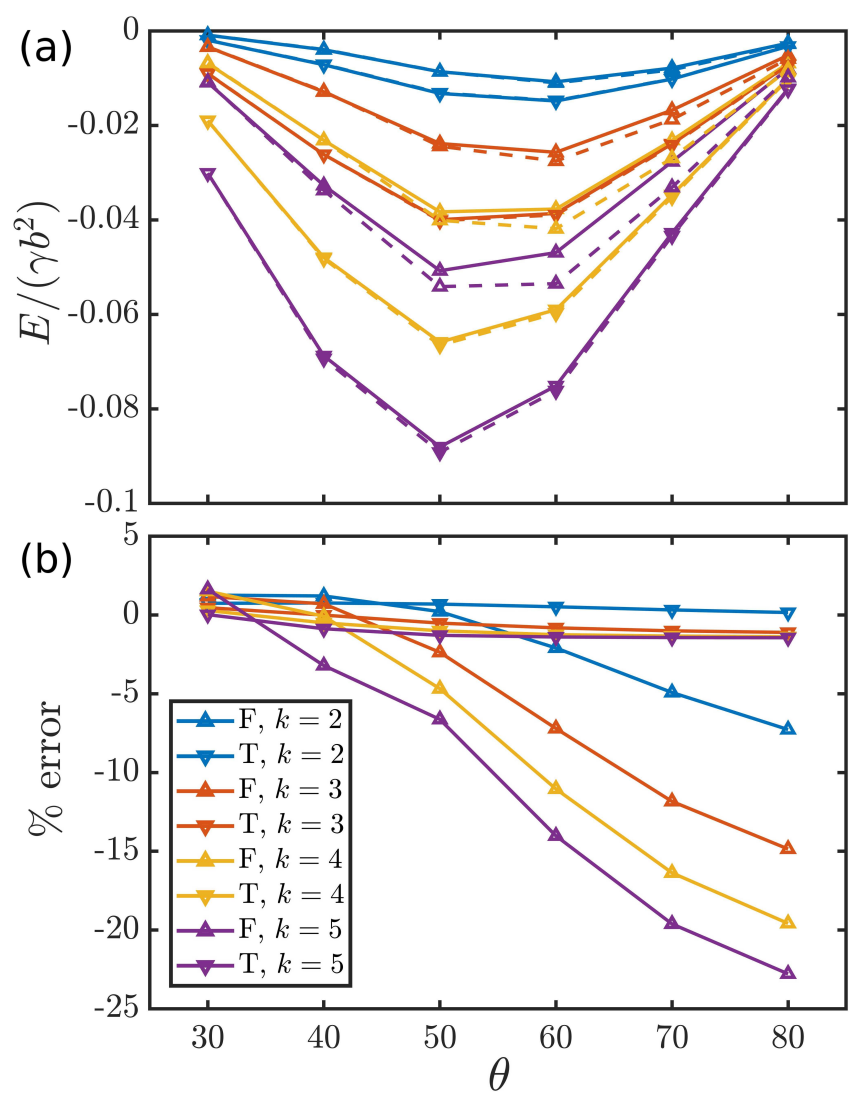

Figure 3: (a) Comparison between the exact three-particle interaction energies (solid lines) and the sum of pairwise interaction energies (dashed lines) for various aspect ratios $k$ and contact angles $\theta$ for particles in the flower (F) and triangle (T) configurations. (b) Percentage relative error (see main text) of the pairwise approximation for the same $k$ and $\theta$ as in the upper panel. 
in Figs. $2 \mathrm{~b}$ and $3 \mathrm{~b}$. As expected, we find that multibody effects are less pro-

\section{Empirical capillary pair potential}

In this Section, we propose a simple empirical pair potential that takes into account the ellipsoidal geometry of our particles, namely by using a scaled distance that is based on the distance of closest approach $d_{\mathrm{ecf}}\left(\phi_{i}, \phi_{j}\right)$ between two was calculated analytically in Ref. [51] and was used in the development of a 'stretched' ellipsoid-shaped Lennard-Jones potential in Ref. [52]. A power law 
fit $\sim 1 / r_{i j}^{m}$ for the capillary interaction energy of ellipsoids with aspect ratio $k=2$ and 3 was attempted in Ref. [37, however, they fitted each configuration (SS, ST, TT) separately and did not investigate a functional form for the angular dependence of the exponent $m$, nor the prefactor.

Our pair potential $U_{i j}$ contains only four fitting parameters and reads

$$
\frac{U_{i j}}{\gamma b^{2}}=-A_{1} \cos \left(2 \phi_{i}+2 \phi_{j}\right)\left(\frac{b}{r_{i j}-A_{2} d_{\mathrm{ecf}}\left(\phi_{i}, \phi_{j}\right)+A_{3}}\right)^{A_{4}},
$$

where $A_{l}$ with $l=1, \ldots, 4$ are the fitting parameters and $r_{i j}$ is the interparticle separation. We can interpret $A_{2}$ as a anisotropy parameter, which characterises the eccentricity of the ellipsoid and $A_{3}$ as an effective particle size. The cosine term reflects the quadrupolar symmetry and ensures that SS and TT interactions are attractive, while the ST interaction is repulsive. Note that the fitting parameters depend on both aspect ratio and contact angle.

The fitting parameters are determined by a least squares fit to the exact two-particle interaction energies in the SS, TT and ST configurations, obtained by Surface Evolver simulations. In line with the physical interpretation of the fitting parameters, we find that $0<A_{2}<1$ and that $a<A_{3}<2 a$. We find $A_{4}$ to be roughly around the far-field value of 4 . The angular accuracy of Eq. (4) is determined by comparison to exact interaction energies in configurations where $r_{i j}$ is fixed and one particle is rotated through $\pi / 2$. We begin the rotation from particles initially in the SS and TT configurations. We fit Eq. (4) to data with $k=2, \ldots, 5$ and $\theta=30^{\circ}, \ldots, 80^{\circ}$. We present a selection of fits for $k=2, \theta=80$ and $k=5, \theta=30$ in Fig. 4. We summarise the values of the fitting parameters in Table 1 .

Looking at the top row of Fig. (4) we see that Eq. (4) lacks the higher order multipoles [21, 33] needed to accurately describe the angular dependence when the ellipsoids are very close together, however, its simplicity and superiority when compared to the quadrupole approximation $(2)$ is clear. In particular, the quadrupole approximation does not distinguish between two particles approaching SS and TT, unlike our empirical pair potential, which can be seen in the 

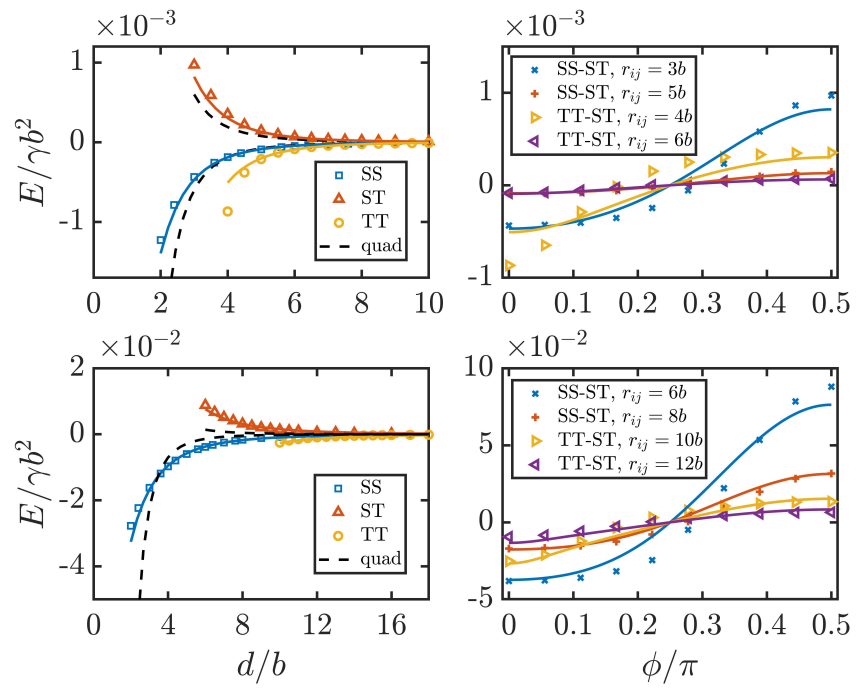

Figure 4: Example fits (solid lines) of our empirical pair potential (4) to the exact SE energies (symbols). We fit according to the SS, ST, TT configurations at different separation distances and a rotation from SS or TT to ST at a fixed separation distance. Upper panels: $k=2$, $\theta=80$. Lower panels: $k=5, \theta=30$. We also show the best fit for the quadrupole approximation (dashed black lines), note that the SS and TT energies are not distinguished by the quadrupole approximation.

$E_{\mathrm{SS}} / E_{\mathrm{TT}}$ is considerably more accurate for our empirical pair potential and we can therefore hope to better model the experimental structures formed by capillary interactions. For $k=2, \theta=50^{\circ}$, the exact Surface Evolver data gives $E_{\mathrm{SS}} / E_{\mathrm{TT}} \approx 3.36$ our empirical pair potential gives $E_{\mathrm{SS}} / E_{\mathrm{TT}} \approx 2.54$, while the quadrupole approximation is off by an order of magnitude $E_{\mathrm{SS}} / E_{\mathrm{TT}} \approx 16.1$. For higher aspect ratios the quadrupole is incorrect by multiple orders of magnitude, which we expect to have a detrimental effect when modelling the self-assembly process.

\section{Self-assembly and structure formation}

We use VMMC simulations to study the self-assembly of ellipsoids interacting via shape-induced capillary interactions using our pair potential in Eq. (4). The VMMC algorithm selects clusters recursively based on differences of interac- 
tion energies, and moves the clusters also according to differences of interaction energies, thereby allowing internal rearrangements within a single cluster. A detailed description of the VMMC algorithm can be found in Refs. [39, 40, 41.

Since the SS and TT bonds between particles in contact are very strong, aggregation is essentially an irreversible process and breaks ergodicity. For this reason we begin with many independent random initial configurations and average over their time evolutions, to imitate experimental procedures where the particles are initially spread onto an interface and then allowed to self-assemble [50]. This procedure is known in the literature as a 'rapid' or 'instantaneous' quench [53, 54, 55].

The aggregation process in dilute colloidal systems is well-studied [56], with two limiting regimes identified: diffusion-limited colloidal aggregation (DLCA), which occurs when the attractive interaction between particles is strong and aggregation is limited by the time taken for clusters to diffuse and encounter each other and reaction-limited colloidal aggregation, which occurs when there is an activation barrier slowing down aggregation. Both cases result in typical fractal cluster structures [57, 58] and scaling laws for the cluster size distribution [59, 60]. There is strong evidence for universality in dilute systems, indicating that the details of the aggregation process are unimportant 61]. We note that the models used to study aggregation are lattice-based 62] or have generally only considered 'sticky' particles [61, which form an infinitely strong bond upon contact so that relaxation of the particles comprising the cluster is not possible.

${ }_{245}$ We expect our capillary dominated system to be more in the DLCA regime, despite the repulsion in certain configurations, because the SS and TT bonds are strong, estimated as $\sim 10^{3} k_{\mathrm{B}} T$.

We investigate the effect of aspect ratio $k$, contact angle $\theta$ and surface coverage $\eta$ on the surface structure formed by capillary interactions. The structures of self-assembled ellipsoids are characterised using the radial pair correlation function $g(r)$ and the angular correlation function $g_{2}(r)=\left\langle\cos 2 \phi_{i j}\right\rangle_{r \pm d r}$, where $\phi_{i j}$ is the angle between particles $i$ and $j$, and their interparticle separation is $(r-d r) \leq r_{i j}<(r+d r)$. For the more dilute surface coverages, we also analyse 
the cluster size distribution $n_{c}\left(c_{s}\right)$, where $c_{s}$ is the number of particles that belong to a cluster (the cluster size) and the number of SS and TT bonds. Whether a particle belongs to a cluster is determined by a geometric criterion, namely if the closest separation distance between two ellipsoids $r_{i j} \leq\left(d_{\mathrm{ecf}}\left(\phi_{i}, \phi_{j}\right)+d r\right)$, where we set the small distance $d r=b / 5$. An SS bond also uses the above geometric criterion, in addition to the angular criterion $\left|\mathbf{u}_{i} \cdot \mathbf{u}_{j}\right|>0.8$, where $\mathbf{u}_{i}$ is the orientation vector of particle $i$. For a TT bond, the angular criteria read $\left|\hat{\mathbf{r}}_{i j} \cdot \mathbf{u}_{i}\right|>0.8$ and $\left|\hat{\mathbf{r}}_{i j} \cdot \mathbf{u}_{j}\right|>0.8$, where $\hat{\mathbf{r}}_{i j}$ is the unit vector connecting particle $i$ to particle $j$. The particle parameters we studied are $k=2-5, \theta=30-80$ and $\eta=0.2-0.6$.

\subsection{Simulation details}

We perform VMMC simulations in a periodic square box with $N=512$ particles. The fitting parameter $A_{1}$ is chosen for computational efficiency such that the TT bond energy is $E_{\mathrm{TT}} \approx 20 k_{\mathrm{B}} T$, which is sufficiently strong that thermal fluctuations are highly unlikely to break the bond. Since the SS capillary bond energy $E_{\mathrm{SS}}$ always seems to be greater than $E_{\mathrm{TT}}$, the SS bond once formed, is also highly unlikely to be broken by thermal noise. Note that by setting the TT bond energy, changing contact angle has the sole effect of changing the ratio between interaction energy at various relative orientations. We employ a cutoff for the capillary interaction at $r_{\text {cut }}=5 a$, which appears reasonable (for not too dilute conditions) given how the interaction strength decays, see Fig. 4. Our empirical potential is then rescaled so that it smoothly reaches zero at $r_{i j}=r_{\text {cut }}$. Random initial configurations are formed by running Metropolis MC using single-particle moves for hard ellipsoids without capillary interactions. 63] We then turn on capillary interactions for the VMMC run. Averages are taken over 20-50 independent runs, with each $10^{5} \mathrm{MC}$ sweeps taking roughly $1.5--2$ hours on an Intel Xeon E5-2680v3 CPU, with the longer times 
relating to higher densities and aspect ratios ${ }^{2}$ The length of the simulations for various surface coverages are summarised in Table 2. Higher surface coverages result in a kinetically arrested state far more quickly than low surface coverages.

In addition to the use of our empirical potential (4) for capillary interactions, we performed simulations using the quadrupole approximation 22 as a comparison (all simulation details are as above). The quadrupole simulations are presented in the supplementary information and turn out to produce slightly different structures than those seen for our empirical potential, which is due to the bond energy ratios $E_{\mathrm{SS}} / E_{\mathrm{TT}}$ for the quadrupole approximation being multiple orders of magnitude too great when compared with the exact Surface Evolver results.

\subsection{Results}

For dilute and moderate surface coverage $\eta \leq 0.4$ we observe that individual particles come together very quickly to form small clusters consisting primarily of chains of SS particles. The chains then come together via their tips to form more isotropic clusters ${ }^{3}$ The aggregation process can be seen for $k=2$ in the top rows of Figs. 5 and for $k=4$ in Fig. 6 .

For the denser systems in the lower rows, the particles very quickly form a spanning cluster and subsequent dynamics are very slow when measured over the simulation timescale, hence we call this a kinetically arrested state. Comparing the bottom panels of Figs. 5 and 6 , we see that at a surface coverage of $\eta=0.7$, the structure formed by the less anisotropic $k=2$ ellipsoids is locally ordered

\footnotetext{
${ }^{2}$ Since our implementation of the VMMC algorithm was not fully optimised, one could even expect improvements in runtime.

${ }^{3}$ For the strong bonds formed by our particles, one would expect the final state to consist of a single spanning cluster. This point is not reached in our simulations with $N=512$ particles due to slowing-down of the aggregation process as the clusters increase in size. We did however perform simulations for smaller systems of $N=128$ and $\eta=0.2$ and we find that to arrive at the final state with one large cluster requires $\sim 5 \times 10^{7} \mathrm{MC}$ sweeps.
} 
but globally disordered. This is to be contrasted with the results for $k=4$ the sterically-stabilised Polymethyl methacrylate (PMMA) ellipsoids of Zhang et al. 64].

\subsubsection{Colloidal aggregation}

We limit discussion here to surface coverages where a spanning cluster is not formed during the simulation $(\eta \leq 0.40)$. Higher surface coverages result very quickly in a kinetically arrested phase and are treated in more detail later. We treat the number of VMMC sweeps $N_{M C}$ as measuring the dynamic evolution of the structure, while keeping in mind that there is not a simple relationship

\footnotetext{
${ }^{4}$ We use the term smectic-like to describe the structure in the snapshot where there is ordering in two directions. Note however, that this structure is kinetically arrested and therefore not a true smectic liquid crystal, which can flow.
} 


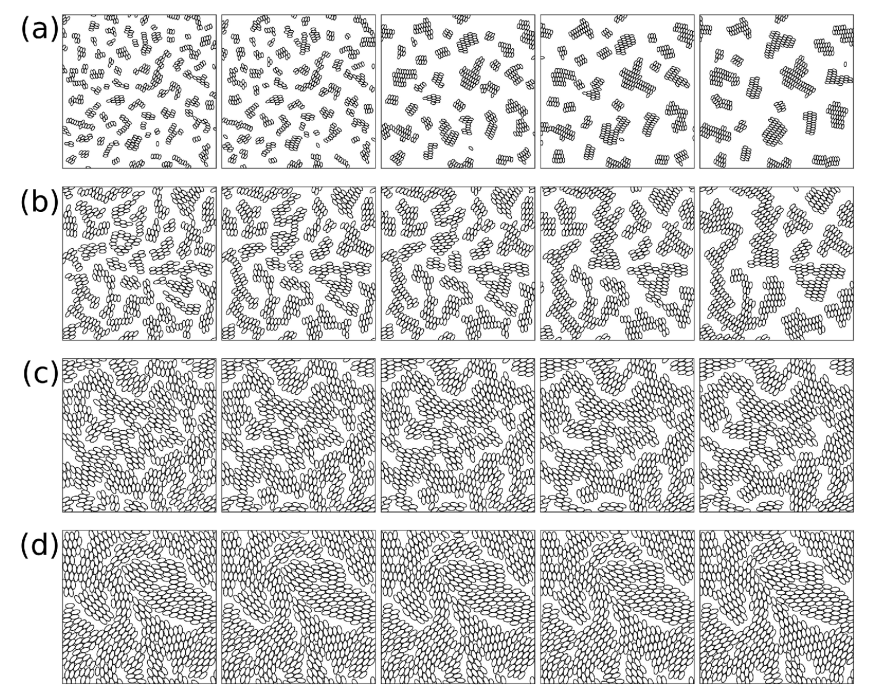

Figure 5: Snapshots of the system showing typical self-assembly and structure evolution of ellipsoidal particles with $k=2, \theta=50$ with different surface coverages. (a) $\eta=0.2$, from left to right, snapshots at VMMC sweeps: $1 \times 10^{4}, 1 \times 10^{5}, 1 \times 10^{6}, 2 \times 10^{6}, 5 \times 10^{6}$. (b) $\eta=0.4$, from left to right, snapshots at VMMC sweeps: $5 \times 10^{3}, 1 \times 10^{4}, 5 \times 10^{4}, 2 \times 10^{5}, 7 \times 10^{5}$. (c) $\eta=0.6$, from left to right, snapshots at VMMC sweeps: $5 \times 10^{3}, 1 \times 10^{4}, 5 \times 10^{4}, 1 \times 10^{5}$, $2 \times 10^{5}$. (d) $\eta=0.7$, from left to right, snapshots at VMMC sweeps: $5 \times 10^{3}, 1 \times 10^{4}, 2 \times 10^{4}$, $5 \times 10^{5}, 1 \times 10^{5}$. 


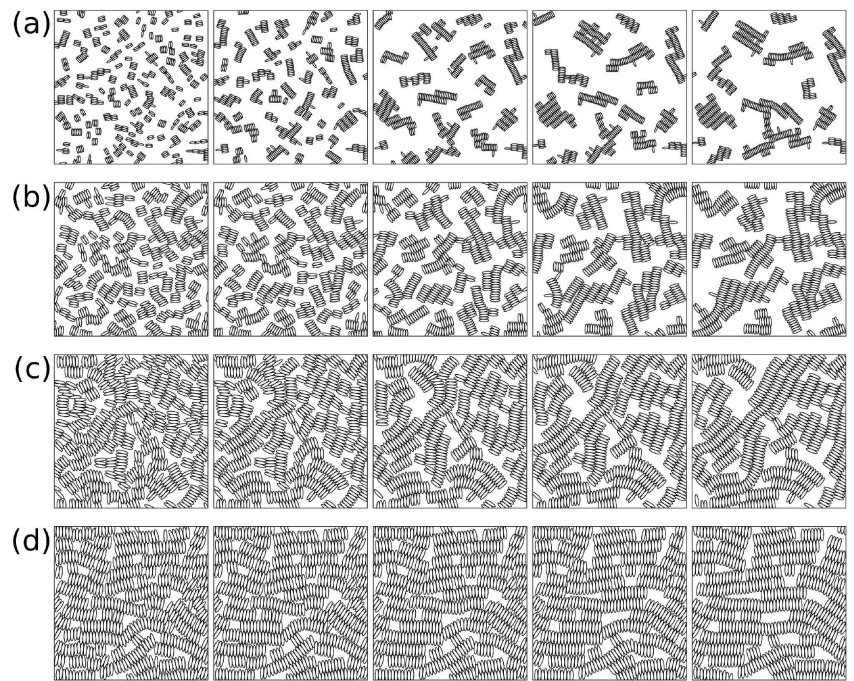

Figure 6: Snapshots of the system showing typical self-assembly and structure evolution of ellipsoidal particles with $k=4, \theta=30$ with different surface coverages. (a) $\eta=0.2$. (b) $\eta=0.4$. (c) $\eta=0.6$. (d) $\eta=0.7$. Snapshots at each surface coverage are taken after the same number of VMMC sweeps as in Fig. 5

between VMMC sweeps and real time $t$. In the context of cluster growth, important time varying quantities are the average cluster size $\left\langle c_{s}(t)\right\rangle$ and the average number of SS and TT bonds $\left\langle n_{\mathrm{SS}}(t)\right\rangle,\left\langle n_{\mathrm{TT}}(t)\right\rangle$, which we present for various aspect ratios, contact angles and surface coverages in Fig. 7. For DLCA, the average cluster size $\left\langle c_{s}(t)\right\rangle$ is predicted to vary as $c_{s}(t) \sim t^{z}$ with dynamic scaling exponent $z$, which is expected to be equal to unity in the dilute limit. 60 , [59] Since we measure time in terms of VMMC sweeps, the value of the exponent $z$ cannot be easily compared with physical time, nevertheless, the underlying power-law behaviour is still apparent and the fact that all data appear to have the same exponent $z$ hints at universality in the aggregation process. In terms of the effect of different particle geometries, we find aggregation to occur faster for higher aspect ratios, and lower contact angles. The effect of $k$ can be understood as the increased probability of more elongated particles to encounter each other. On the other hand, the effect of $\theta$ is to change the SS bond energy (since we fix $\left.E_{\mathrm{TT}}\right)$ and since smaller contact angles have a greater ratio $E_{\mathrm{SS}} / E_{\mathrm{TT}}$, the 

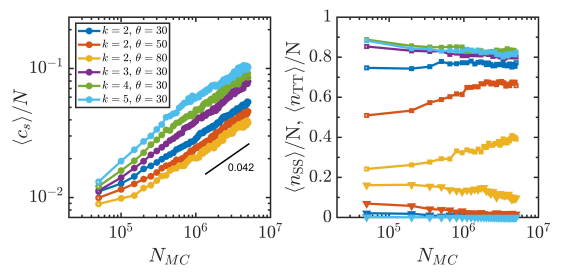

Figure 7: Left panel: Evolution of the average cluster size $\left\langle c_{s}\right\rangle$ for various aspect ratios, contact angles and surface coverages in a log-log plot. Black line shows a power law with exponent 0.042. Right panel: Average number of SS bonds $\left\langle n_{\mathrm{SS}}\right\rangle$ (squares), and average number of TT bonds $\left\langle n_{\mathrm{TT}}\right\rangle$ (triangles). Colours correspond to the same parameters in the left panel.

attractive SS interaction is stronger resulting in a higher average number of SS bonds compared to TT bonds for smaller contact angles. We observe from snapshots of the simulation in Figs. 5 and 6 that smaller clusters come together to form larger clusters. This process results in the number of smaller clusters decreasing and a growth in the number of larger clusters, as can be clearly seen in the evolution of the cluster size distribution in Fig. 8 for one set of parameters. The universal behaviour of DLCA is manifested in the cluster size distribution, which can be collapsed onto a master curve using the scaling law [60, 61, 62

$$
\frac{n_{s}}{N} \sim\left\langle c_{s}(t)\right\rangle^{-2} f\left(\frac{c_{s}}{\left\langle c_{s}(t)\right\rangle}\right)
$$

where $f$ is a universal bell-shaped function. We show the cluster size distribution dynamics in Fig. 8 and in the inset of Fig. 8, demonstrate data collapse onto a master curve using the scaling law (5). We note that data collapse is observed across all aspect ratios and contact angles for $\eta \leq 0.4$, which is quite interesting given that the scaling law is in principle, only valid for dilute systems. The cluster-size dynamics we observe indicate that the aggregation of colloids by capillary interaction fall into the universality class of DLCA.

\subsubsection{High density}

We now proceed with a more quantitative analysis of the structures encountered in our high surface coverage states beginning with the radial and angular correlation functions, which highlight the effect that anisotropy and particle ge- 


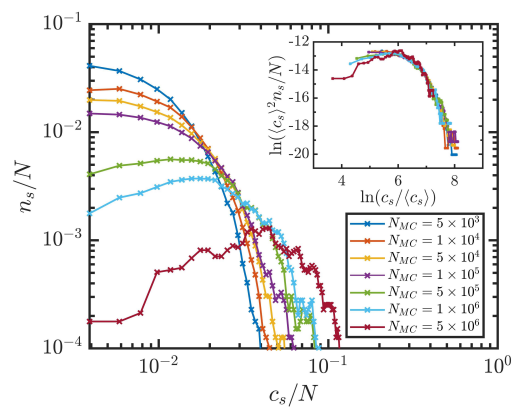

Figure 8: The cluster size distribution $n_{s}\left(c_{s}\right)$ for $k=2, \theta=50, \eta=0.2$ at different times. Cluster-cluster aggregation shifts the distribution towards larger cluster sizes for later times. Inset: Scaling behaviour of the cluster size distribution showing the collapse of data in the main plot onto a master curve according to Eq. [5].

ometry have on the final structure. Fig. 9 shows pair correlation functions $g(r)$ and $g_{2}(r)$ for $k=2$ and $k=4$ particles at a surface coverage $\eta=0.6$. We see the largest peak in $g(r)$ at $r=2 b$. Further peaks follow at multiples of $2 b$ and $2 a$, corresponding to the SS and TT configurations respectively. The smaller peaks of $g(r)$ and $g_{2}(r)$ just before $r=4 b$ for $k=2$ and $r=8 b$ for $k=4$ (indicated by arrows in Fig. 99 is due to the ellipsoids interlocking at the tips in a crystalline structure, like zipper teeth.

We see from the plots of $g_{2}(r)$ that the angular correlations are strongest when $r$ is multiples of $2 b$ and $2 a$ and also that they decay slowly, indicating long-range orientational correlations. We also see that particle geometry plays a role in the characteristics of the final structures. Namely that for higher aspect ratios, the angular correlations decay more slowly and the peaks are more pronounced. These characteristics can be explained by the fact that higher aspect ratios cause chains of SS particles to be more rigid, as is seen in the bottom rows of Figs. 5 and 6 , where $k=2$ ellipsoids form 'bendier' structures when interlocked at the tips than the straight structures favoured by the more elongated $k=4$ ellipsoids. Indeed, the peaks corresponding to chains of $k=4$ SS particles are evident in Fig. 9 The additional rigidity is not evident in the dilute regime since there are fewer constraints on the particle chains so that the 
$k=2$ ellipsoid chains are free to straighten.
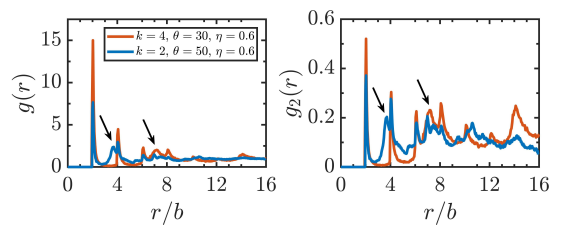

Figure 9: Typical radial and angular correlation functions for high density kinetically arrested states. Arrows indicate the separation distances where the tips are interlocked like zipper teeth.

355

\subsection{Comparison to experiments}

We compare the simulation results with experimental structure observations of sterically-stabilised PMMA ellipsoids. These were prepared according to the well-established procedure in Zhang et al. 64, by stretching 3 micron PMMA pect ratio $k \approx 2$ and $k \approx 4-5$, respectively. Such particles are expected to effectively model hard-core interactions. The particles were labelled with small amounts of organic fluorescent dye 7-nitrobenzo-2-oxa-1,3-diazol to facilitate their observation at a fluid interface, for which we used an inverted fluorescence microscope (Nikon TI-2).

For identically prepared PMMA ellipsoids, the contact angle is a function of the aspect ratio. 65. These contact angles at the single particle level were measured using FRESCA [66] however, this method is limited to oil-water interfaces. The contact angle increased from $103 \pm 15^{\circ}$ degrees for $k \approx 2$ to $113 \pm 7^{\circ}$ degrees for the simulations in the range of $\theta=30-50^{\circ}$. The particles were dispersed in hexane (1\% by weight) and approximately $20 \mu \mathrm{L}$ was deposited onto a planar decalin-air interface, created using sample cells (diameter of $2 \mathrm{~cm}$ ) which pinned the contact line of the oil. These sample cells could be closed which, combined with the high boiling point of decalin, enabled long term experiments. During spreading, Marangoni stresses lead to rather strong surface flows, after which 
the interface comes to rest. For $k \approx 4$ a dense interface was created by spreading from a dense suspension. For the experiments with $k \approx 2$ a more dilute initial state was used and the structure was observed to become denser over time. The structures were imaged after 48 hours.

We show experimental observations and simulated structures side by side in Fig. 10, where we see that our empirical pair potential and the pairwise approximation seem sufficient to reproduce the experimental observations. There is a clear similarity at relatively high surface coverages, from the smectic-like structure of the densely packed $k=4$ ellipsoids to the more disordered gel-like structure of the $k=2$ ellipsoids. One should, however, bear in mind that since the particles are very strongly attractive, the system is non-ergodic and hence the surface structures depend to some degree on the kinetic pathways. Whether our simulation protocol truly represents the self-assembly process remains an open question.

\section{Conclusion}

We showed that the multibody capillary interaction for three particles can be reasonably described by pairwise interaction energies and proposed a simple yet accurate empirical form for the pairwise shape-induced capillary interaction between ellipsoidal particles that is far superior to the quadrupole approximation [33. Thanks to these first steps, we were able to efficiently model the structure formation process with hundreds of particles in a Monte-Carlo type simulations and verify our approach by making direct comparisons to our experimental results, particularly at high surface coverages where excluded volume and capillary interactions dominate. In comparison to previous literature, we can efficiently simulate hundreds of particles, rather than the handful possible in Surface Evolver simulations 35, 36, 37. Furthermore, compared with the Lattice-Boltzmann simulations of Harting et al. 42, 43, 44, we treated the capillary interactions more accurately. Our approach clarifies the relevant param- 


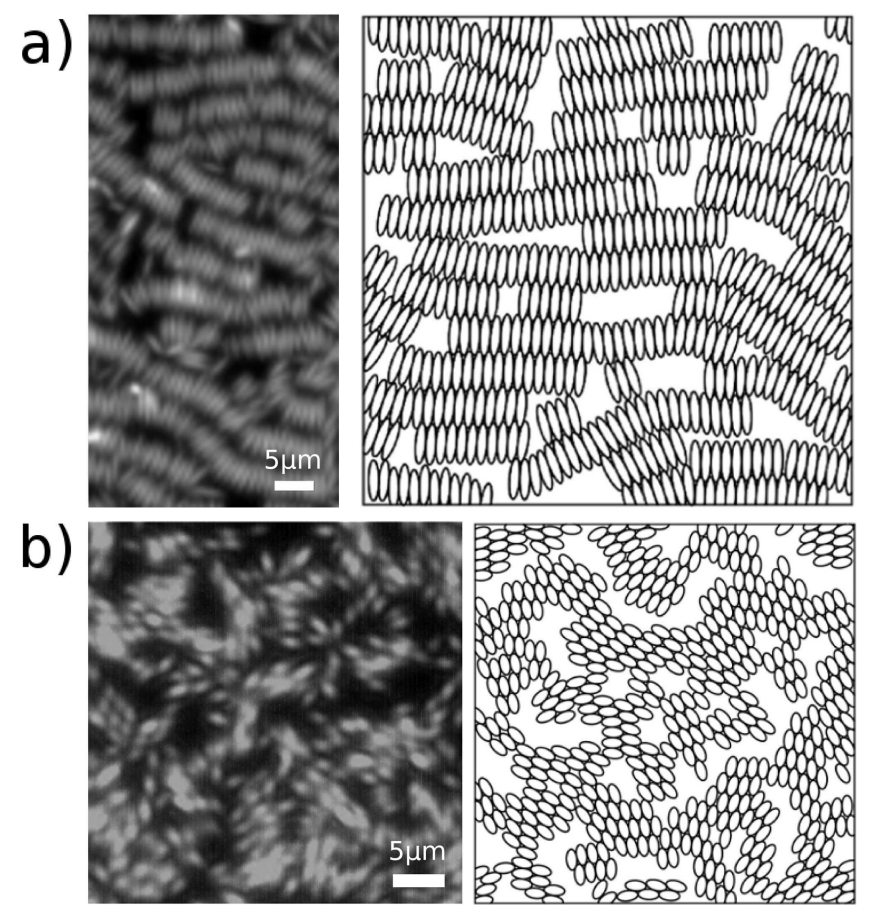

Figure 10: Side by side comparison between experimental observations of surface structure (left) and simulations (right). a) $k \approx 4$ PMMA sterically-stabilised ellipsoidal particles at an oil-air interface after 48 hours compared with simulations of $k=4, \theta=30, \eta=0.7$ ellipsoids after $2 \times 10^{5}$ sweeps. b) $k \approx 2$ PMMA sterically-stabilised ellipsoidal particles at an oil-air interface 48 hours after spreading compared with simulations of $k=2, \theta=50, \eta=0.6$ ellipsoids after $5 \times 10^{4}$ sweeps.

coverage and to a far lesser extent, contact angle.

Further work including other relevant interactions such as electrostatic interactions is needed to fully account for the whole range of structures. Having established an approximate pair potential that extends Eq. (4) to include these other relevant interactions allows a more thorough investigation into the structure formation, phase behavior and dynamical properties along the lines explored in Luo et al.67]. In addition, we could extend our modelling procedure to allow for ellipsoids that tilt out of the interface, which has been experimentally observed by compressing a surface monolayer 68] and computationally modelled with magnetic particles in a magnetic field [69, 170]. 


\section{Conflicts of interest}

There are no conflicts to declare.

\section{Acknowledgements}

We would like to thank Victoria Blair for her help with the experiments as well as Hans Christian Öttinger for critically reading and offering suggestions to improve the manuscript. Patrick Ilg was also supported by EU FP7-MC-CIG Grant No. 631233.

\section{References}

[1] A. Striolo, J. Kim, C. Murphy, L. Liz-Marzán, J. Lahann, J. Reguera, Y. Zhou, M. Brust, A. Thill, L. Scarabelli, T. A. F. König, M. Buzza, C. Kuttner, E. G. Solveyra, H. Wolf, J. Vermant, M. Pauly, A. Harvie, L. Pasquato, A. Stocco, H. Mattoussi, E. Kumacheva, K. Heatley, C. Hanske, R. Faller, D. French, A. Honciuc, B. Binks, F. Sicard, Particles at interfaces: general discussion, Faraday Discuss. 191 (0) (2016) 407-434. doi:10.1039/C6FD90050J. URL http://pubs.rsc.org/en/content/articlelanding/2016/fd/ c6fd90050j

[ [2] W. Ramsden, Separation of Solids in the Surface-Layers of Solutions and

1. 'Suspensions' (Observations on Surface-Membranes, Bubbles, Emulsions, and Mechanical Coagulation). - Preliminary Account, Proc. R. Soc. Lond. 72 (477-486) (1903) 156-164. doi:10.1098/rspl.1903.0034. URL http://rspl.royalsocietypublishing.org/content/72/ $477-486 / 156$

[3] S. U. Pickering, CXCVI.-Emulsions, J. Chem. Soc., Trans. 91 (0) (1907) 2001-2021. doi:10.1039/CT9079102001.

口 URL http://pubs.rsc.org/en/content/articlelanding/1907/ct/ ct9079102001 
[ [4] G. G. Fuller, J. Vermant, Complex Fluid-Fluid Interfaces: Rheology and Structure, Annual Review of Chemical and Biomolecular Engineering 3 (1) (2012) 519-543. doi:10.1146/annurev-chembioeng-061010-114202 URL https://doi .org/10.1146/annurev-chembioeng-061010-114202

q [5] J. Wu, G.-H. Ma, Recent Studies of Pickering Emulsions: Particles Make the Difference, Small 12 (34) (2016) 4633-4648. doi:10.1002/smll.201600877.

450 URL http://onlinelibrary.wiley.com/doi/10.1002/smll. 201600877/abstract

[6] B. P. Binks, T. S. Horozov, Colloidal Particles at Liquid Interfaces, Cambridge University Press, 2008.

q [7] S. Sacanna, W. K. Kegel, A. P. Philipse, Thermodynamically Stable Pick455 ㅁ ering Emulsions, Phys. Rev. Lett. 98 (15) (2007) 158301. doi:10.1103/ PhysRevLett.98.158301. URL https://link.aps.org/doi/10.1103/PhysRevLett.98.158301

[8] P. J. Beltramo, M. Gupta, A. Alicke, I. Liascukiene, D. Z. Gunes, C. N. Baroud, J. Vermant, Arresting dissolution by interfacial rheology design, PNAS 114 (39) (2017) 10373-10378. doi:10.1073/pnas.1705181114. URL http://www . pnas .org/content/114/39/10373

n [9] E. Vignati, R. Piazza, T. P. Lockhart, Pickering Emulsions: Interfacial Tension, Colloidal Layer Morphology, and Trapped-Particle Motion, Langmuir 19 (17) (2003) 6650-6656. doi:10.1021/la0342641. URL http://dx.doi.org/10.1021/la0342641

[10] A. Stocco, W. Drenckhan, E. Rio, D. Langevin, B. P. Binks, Particlestabilised foams: an interfacial study, Soft Matter 5 (11) (2009) 2215-2222. doi:10.1039/B901180C.

a URL http://pubs.rsc.org/en/content/articlelanding/2009/sm/ 470 b901180c 
[11] P. Pieranski, Two-dimensional interfacial colloidal crystals, Phys. Rev. Lett. 45 (1980) 569-572. doi:10.1103/PhysRevLett.45.569. URL https://link .aps.org/doi/10.1103/PhysRevLett.45.569

[12] A. J. Hurd, The electrostatic interaction between interfacial colloidal par475 ticles, J. Phys. A: Math. Gen. 18 (16) (1985) L1055. doi:10.1088/ 0305-4470/18/16/011

URL http://stacks.iop.org/0305-4470/18/i=16/a=011

[13] R. Aveyard, B. P. Binks, J. H. Clint, Emulsions stabilised solely by colloidal particles, Advances in Colloid and Interface Science 100 (Supplement C) (2003) 503-546. doi:10.1016/S0001-8686(02)00069-6.

प URL http://www.sciencedirect.com/science/article/pii/ S0001868602000696

[14] K. Masschaele, S. Vandebril, J. Vermant, B. Madivala, Interfacial Rheology, in: C. Gallegos (Ed.), Rheology - Volume II, EOLSS Publications, 2010.

${ }_{485}[15]$ P. A. Kralchevsky, K. Nagayama, Capillary interactions between particles bound to interfaces, liquid films and biomembranes, Advances in Colloid and Interface Science 85 (2) (2000) 145-192. doi:10.1016/S0001-8686(99)00016-0.

a URL http://www.sciencedirect.com/science/article/pii/

490 S0001868699000160

[16] J. C. Loudet, A. G. Yodh, B. Pouligny, Wetting and Contact Lines of 1 Micrometer-Sized Ellipsoids, Phys. Rev. Lett. 97 (1) (2006) 018304. doi: 10.1103/PhysRevLett.97.018304.

URL https://link.aps.org/doi/10.1103/PhysRevLett.97.018304

[17] D. Vella, L. Mahadevan, The "Cheerios effect", American Journal of Physics 73 (9) (2005) 817-825. doi:10.1119/1.1898523.

URL http://aapt.scitation.org/doi/10.1119/1.1898523 
[18] D. Y. C. Chan, J. D. Henry, L. R. White, The interaction of colloidal particles collected at fluid interfaces, Journal of Colloid and Interface Science 79 (2) (1981) 410-418. doi:10.1016/0021-9797(81)90092-8. URL http://www.sciencedirect.com/science/article/pii/ 0021979781900928

[19] J. C. Loudet, B. Pouligny, How do mosquito eggs self-assemble on the water surface? Eur. Phys. J. $\quad$ E 34 (8) (2011) 76. doi:10.1140/epje/i2011-11076-9.

a URL https://link.springer.com/article/10.1140/epje/ i2011-11076-9

[20] G. Soligno, M. Dijkstra, R. van Roij, Self-Assembly of Cubes into 2d Hexagonal and Honeycomb Lattices by Hexapolar Capillary Interactions,

$510 \quad$ Phys. Rev. Lett. 116 (25) (2016) 258001. doi:10.1103/PhysRevLett.116. 258001.

URL https://link.aps .org/doi/10.1103/PhysRevLett.116.258001

[21] D. Stamou, C. Duschl, D. Johannsmann, Long-range attraction between a colloidal spheres at the air-water interface: The consequence of an irregular 515 meniscus, Phys. Rev. E 62 (4) (2000) 5263-5272. doi:10.1103/PhysRevE. 62.5263

URL https://link.aps.org/doi/10.1103/PhysRevE.62.5263

[22] R. V. Hooghten, V. E. Blair, A. Vananroye, A. B. Schofield, J. Vermant,

п J. H. J. Thijssen, Interfacial Rheology of Sterically Stabilized Colloids 520 at Liquid Interfaces and Its Effect on the Stability of Pickering Emulq Sions, Langmuir 33 (17) (2017) 4107-4118. doi:10.1021/acs.langmuir. $6 \mathrm{~b} 04365$

URL http://dx.doi.org/10.1021/acs . langmuir.6b04365

[23] M. Zanini, C. Marschelke, S. E. Anachkov, E. Marini, A. Synytska, ${ }_{525}$ a L. Isa, Universal emulsion stabilization from the arrested adsorption of rough particles at liquid-liquid interfaces, Nat Commun 8 (2017) 15701. 
doi:10.1038/ncomms15701.

URL https://www.ncbi.nlm.nih.gov/pmc/articles/PMC5467241/

[24] M. Oettel, S. Dietrich, Colloidal Interactions at Fluid Interfaces, Langmuir 24 (4) (2008) 1425-1441. doi:10.1021/la702794d.

URL http://dx .doi .org/10.1021/la702794d

[25] K. D. Danov, P. A. Kralchevsky, M. P. Boneva, Shape of the Capillary

a Meniscus around an Electrically Charged Particle at a Fluid Interface: Comparison of Theory and Experiment, Langmuir 22 (6) (2006) 26532667. doi:10.1021/la052749z.

URL http://dx .doi.org/10.1021/la052749z

[26] M. Girotto, A. P. dos Santos, Y. Levin, Interaction of Charged Colloidal Particles at the Air-Water Interface, J. Phys. Chem. B 120 (26) (2016) 5817-5822. doi:10.1021/acs.jpcb.5b10105. URL http://dx .doi .org/10.1021/acs .jpcb.5b10105

[27] R. Aveyard, J. H. Clint, D. Nees, V. N. Paunov, Compression and Structure 1 of Monolayers of Charged Latex Particles at Air/Water and Octane/Water Interfaces, Langmuir 16 (4) (2000) 1969-1979. doi:10.1021/la990887g. URL http://dx .doi .org/10.1021/la990887g

[28] R. Aveyard, B. P. Binks, J. H. Clint, P. D. I. Fletcher, T. S. Horozov, B. Neumann, V. N. Paunov, J. Annesley, S. W. Botchway, D. Nees, A. W. Parker, A. D. Ward, A. N. Burgess, Measurement of Long-Range Repulsive Forces between Charged Particles at an Oil-Water Interface, Phys. Rev. Lett. 88 (24) (2002) 246102. doi:10.1103/PhysRevLett.88.246102.

[29] J. C. Loudet, A. M. Alsayed, J. Zhang, A. G. Yodh, Capillary Interactions Between Anisotropic Colloidal Particles, Phys. Rev. Lett. 94 (1) (2005) 018301. doi:10.1103/PhysRevLett.94.018301.

URL https : //link .aps .org/doi/10.1103/PhysRevLett.94.018301 
- Direct Measurements of the Effects of Salt and Surfactant on Interaction Forces between Colloidal Particles at Water-Oil Interfaces, Langmuir 24 (5)

(2008) 1686-1694. doi:10.1021/la7008804.

URL http://dx.doi .org/10.1021/la7008804

560

[31] K. D. Danov, P. A. Kralchevsky, Capillary forces between particles at a

1 liquid interface: General theoretical approach and interactions between capillary multipoles, Advances in Colloid and Interface Science 154 (1)

(2010) 91-103. doi:10.1016/j.cis.2010.01.010.

565

[32] A. Domínguez, M. Oettel, S. Dietrich, Force balance of particles trapped

1. at fluid interfaces, J. Chem. Phys. 128 (11) (2008) 114904. doi:10.1063/ 1.2890035 .

URL http://aip.scitation.org/doi/10.1063/1.2890035

[33] H. Lehle, E. Noruzifar, M. Oettel, Ellipsoidal particles at fluid inter-

1] faces, Eur. Phys. J. E 26 (1-2) (2008) 151-160. doi:10.1140/epje/ i2007-10314-1.

URL http://dx.doi.org/10.1140/epje/i2007-10314-1

[34] J.-B. Fournier, P. Galatola, Anisotropic capillary interactions and jamming of colloidal particles trapped at a liquid-fluid interface, Phys. Rev. E 65 (3) (2002) 031601. doi:10.1103/PhysRevE.65.031601

URL https : //link.aps.org/doi/10.1103/PhysRevE.65.031601

[35] L. Botto, L. Yao, R. L. Leheny, K. J. Stebe, Capillary bond between rod-like particles and the micromechanics of particle-laden interfaces, Soft

580 Matter 8 (18) (2012) 4971-4979. doi:10.1039/C2SM25211B. c2sm25211b 
[36] L. Botto, E. P. Lewandowski, M. Cavallaro, K. J. Stebe, Capillary interactions between anisotropic particles, Soft Matter 8 (39) (2012) 9957-9971. doi:10.1039/C2SM25929J.

口 URL http://pubs.rsc.org/en/content/articlelanding/2012/sm/ c2sm25929j

[37] S. Dasgupta, M. Katava, M. Faraj, T. Auth, G. Gompper, Capillary Asa sembly of Microscale Ellipsoidal, Cuboidal, and Spherical Particles at Interfaces, Langmuir 30 (40) (2014) 11873-11882. doi:10.1021/la502627h URL http://dx.doi.org/10.1021/la502627h

[38] P. Galatola, Capillary force and torque on spheroidal particles floating at a fluid interface beyond the superposition approximation, Phys Rev E 93 (2) (2016) 022604. doi:10.1103/PhysRevE.93.022604

[39] S. Whitelam, P. L. Geissler, Avoiding unphysical kinetic traps in Monte Carlo simulations of strongly attractive particles, The Journal of Chemical Physics 127 (15) (2007) 154101. doi:10.1063/1.2790421.

URL http://aip.scitation.org/doi/10.1063/1.2790421

[40] Š. Růžička, M. P. Allen, Collective translational and rotational Monte Carlo cluster move for general pairwise interaction, Phys. Rev. E 90 (3) (2014) 033302. doi:10.1103/PhysRevE.90.033302

URL https://link.aps.org/doi/10.1103/PhysRevE.90.033302

[41] Š. Růžička, M. P. Allen, Collective translational and rotational Monte Carlo 1. moves for attractive particles, Phys. Rev. E 89 (3) (2014) 033307. doi: 10.1103/PhysRevE.89.033307. URL https://link.aps.org/doi/10.1103/PhysRevE.89.033307

[42] F. Günther, S. Frijters, J. Harting, Timescales of emulsion formation caused by anisotropic particles, Soft Matter 10 (27) (2014) 4977-4989. doi:10.1039/C3SM53186D,

610 URL http://pubs.rsc.org/en/content/articlelanding/2014/sm/ c3sm53186d 
[43] T. Krüger, S. Frijters, F. Günther, B. Kaoui, J. Harting, Numerical simulations of complex fluid-fluid interface dynamics, Eur. Phys. J. Spec.

Top. 222 (1) (2013) 177-198. doi:10.1140/epjst/e2013-01834-y. e2013-01834-y

[44] F. Jansen, J. Harting, From bijels to Pickering emulsions: A lattice Boltz-

1. mann study, Phys. Rev. E 83 (4) (2011) 046707. doi:10.1103/PhysRevE. 83.046707 . URL https://link .aps .org/doi/10.1103/PhysRevE.83.046707

[45] M. M. Nicolson, The interaction between floating particles, Mathematical Proceedings of the Cambridge Philosophical Society 45 (2) (1949) 288-295. doi:10.1017/S0305004100024841.

1 URL https://www.cambridge.org/core/journals/ mathematical-proceedings-of-the-cambridge-philosophical-society/

[46] M. Oettel, A. Domínguez, S. Dietrich, Effective capillary interaction of spherical particles at fluid interfaces, Phys. Rev. E 71 (5) (2005) 051401.

${ }_{630}$ doi:10.1103/PhysRevE.71.051401

URL https://link . aps .org/doi/10.1103/PhysRevE.71.051401

[47] N. D. Vassileva, D. van den Ende, F. Mugele, J. Mellema, Capillary Forces between Spherical Particles Floating at a Liquid-Liquid Interface, Langmuir 21 (24) (2005) 11190-11200. doi:10.1021/la0511860. URL http://dx.doi.org/10.1021/la0511860

[48] A. Domínguez, M. Oettel, S. Dietrich, Theory of capillary-induced interactions beyond the superposition approximation, J. Chem. Phys. 127 (20) (2007) 204706. doi:10.1063/1.2781420.

URL http://aip.scitation.org/doi/abs/10.1063/1.2781420 
[51] X. Zheng, P. Palffy-Muhoray, Distance of closest approach of two arbitrary

口 hard ellipses in two dimensions, Phys. Rev. E 75 (6) (2007) 061709. doi: 10.1103/PhysRevE.75.061709.

U50 URL https://link.aps.org/doi/10.1103/PhysRevE.75.061709

[52] L. Paramonov, S. N. Yaliraki, The directional contact distance of two ellipsoids: Coarse-grained potentials for anisotropic interactions, The Journal of Chemical Physics 123 (19) (2005) 194111. doi:10.1063/1.2102897. URL http://aip.scitation.org/doi/full/10.1063/1.2102897

[53] J. C. F. Toledano, F. Sciortino, E. Zaccarelli, Colloidal systems with competing interactions: from an arrested repulsive cluster phase to a gel Soft Matter 5 (12) (2009) 2390-2398. doi:10.1039/B818169A

11 URL http://pubs.rsc.org/en/content/articlelanding/2009/sm/ b818169a

[54] A. Malins, J. Eggers, H. Tanaka, C. P. Royall, Lifetimes and lengthscales of structural motifs in a model glassformer, Faraday Discuss. 167 (0) (2014) 405-423. doi:10.1039/C3FD00078H

口

URL http://pubs.rsc.org/en/content/articlelanding/2013/fd/ c3fd00078h

[55] Š. Růžička, M. P. Allen, Monte Carlo simulation of kinetically slowed down phase separation, Eur. Phys. J. E 38 (6) (2015) 68. doi:10.1140/epje/i2015-15068-5 
URL

https://link.springer.com/article/10.1140/epje/ i2015-15068-5

[60] M. Kolb, R. Botet, R. Jullien, Scaling of Kinetically Growing Clusters

URL https://link.aps.org/doi/10.1103/PhysRevLett.51.1123

[61] A. E. González, Universality of colloid aggregation in the reaction limit:

n The computer simulations, Phys. Rev. Lett. 71 (14) (1993) 2248-2251. doi:

$690 \quad 10.1103 /$ PhysRevLett.71.2248.

URL https://link.aps.org/doi/10.1103/PhysRevLett.71.2248

[62] M. Lach-hab, A. E. González, E. Blaisten-Barojas, Concentration de-

п pendence of structural and dynamical quantities in colloidal aggregation:

a Computer simulations, Phys. Rev. E 54 (5) (1996) 5456-5462. doi: 
URL https://link.aps.org/doi/10.1103/PhysRevE.54.5456

[63] A. M. Luo, L. M. C. Sagis, P. Ilg, The Landau free energy of hard ellipses obtained from microscopic simulations, The Journal of Chemical Physics 140 (12) (2014) 124901. doi:10.1063/1.4868988.

URL http://aip.scitation.org/doi/full/10.1063/1.4868988

[64] Z. Zhang, P. Pfleiderer, A. B. Schofield, C. Clasen, J. Vermant, Synthesis and Directed Self-Assembly of Patterned Anisometric Polymeric Particles, J. Am. Chem. Soc. 133 (3) (2011) 392-395. doi:10.1021/ja108099r.

URL http://dx.doi.org/10.1021/ja108099r

705 [65] S. Coertjens, P. Moldenaers, J. Vermant, L. Isa, Contact Angles of Microellipsoids at Fluid Interfaces, Langmuir 30 (15) (2014) 4289-4300. doi:10.1021/la500888u.

URL http://dx.doi.org/10.1021/la500888u

[66] L. Isa, F. Lucas, R. Wepf, E. Reimhult, Measuring single-nanoparticle wet$710 \quad$ 口 ting properties by freeze-fracture shadow-casting cryo-scanning electron microscopy, Nat Commun 2 (2011) 438. doi:10.1038/ncomms1441. URL http://www.ncbi.nlm.nih.gov/pmc/articles/PMC3265365/

[67] A. M. Luo, L. M. C. Sagis, H. C. Öttinger, C. D. Michele, P. Ilg, Modelling

1. the rheology of anisotropic particles adsorbed on a two-dimensional fluid in715 terface, Soft Matter 11 (22) (2015) 4383-4395. doi:10.1039/C5SM00372E

a URL http://pubs.rsc.org/en/content/articlelanding/2015/sm/ c5sm00372e

[68] M. G. Basavaraj, G. G. Fuller, J. Fransaer, J. Vermant, Packing, Flipping, 1 a and Buckling Transitions in Compressed Monolayers of Ellipsoidal Latex ${ }_{720}$ Particles, Langmuir 22 (15) (2006) 6605-6612. doi:10.1021/1a060465o. URL http://dx.doi.org/10.1021/la060465o 
[69] G. B. Davies, T. Krüger, P. V. Coveney, J. Harting, F. Bresme, As-

n sembling Ellipsoidal Particles at Fluid Interfaces Using Switchable Dipolar Capillary Interactions, Adv. Mater. 26 (39) (2014) 6715-6719. URL http://onlinelibrary.wiley.com/doi/10.1002/adma. 201402419/abstract

[70] G. B. Davies, L. Botto, Dipolar capillary interactions between tilted ellipsoidal particles adsorbed at fluid-fluid interfaces, Soft Matter 11 (40) (2015) 7969-7976. doi:10.1039/C5SM01815C

a URL http://pubs.rsc.org/en/content/articlelanding/2015/sm/ c5sm01815c 
Table 1: Parameters for our empirical pair potential for a range of $k$ and $\theta$, determined by fitting to precise numerical simulation data. Fit uncertainties are within approximately $10 \%$ of the stated values.

\begin{tabular}{|c|c|c|c|c|c|}
\hline & $\theta$ & $A_{1}$ & $A_{2}$ & $A_{3}$ & $A_{4}$ \\
\hline \multirow{6}{*}{$k=2$} & 30 & 1.030 & 0.313 & 2.771 & 4.806 \\
\hline & 40 & 1.591 & 0.433 & 2.505 & 4.722 \\
\hline & 50 & 1.414 & 0.603 & 2.536 & 4.538 \\
\hline & 60 & 1.112 & 0.715 & 2.662 & 4.418 \\
\hline & 70 & 0.859 & 0.748 & 2.776 & 4.479 \\
\hline & 80 & 1.217 & 0.356 & 2.713 & 4.511 \\
\hline \multirow{6}{*}{$k=3$} & 30 & 4.455 & 0.350 & 2.895 & 4.511 \\
\hline & 40 & 4.087 & 0.497 & 2.967 & 4.024 \\
\hline & 50 & 3.797 & 0.637 & 3.109 & 3.864 \\
\hline & 60 & 3.215 & 0.733 & 3.247 & 3.859 \\
\hline & 70 & 3.189 & 0.786 & 3.572 & 4.074 \\
\hline & 80 & 2.435 & 0.801 & 4.018 & 4.435 \\
\hline \multirow{6}{*}{$k=4$} & 30 & 16.33 & 0.331 & 3.821 & 4.192 \\
\hline & 40 & 16.35 & 0.523 & 3.558 & 3.983 \\
\hline & 50 & 16.37 & 0.644 & 4.397 & 3.726 \\
\hline & 60 & 16.37 & 0.726 & 4.671 & 3.786 \\
\hline & 70 & 16.37 & 0.767 & 5.085 & 3.984 \\
\hline & 80 & 16.36 & 0.783 & 5.870 & 4.391 \\
\hline \multirow{6}{*}{$k=5$} & 30 & 20.01 & 0.394 & 4.086 & 3.851 \\
\hline & 40 & 19.80 & 0.501 & 4.762 & 3.445 \\
\hline & 50 & 19.41 & 0.602 & 5.112 & 3.339 \\
\hline & 60 & 19.32 & 0.668 & 5.534 & 3.383 \\
\hline & 70 & 17.58 & 0.703 & 6.061 & 3.528 \\
\hline & 80 & 17.38 & 0.717 & 7.049 & 3.915 \\
\hline
\end{tabular}


Table 2: Summary of the total number of MC sweeps for different surface coverages $\eta$. An MC sweep is defined as $N$ attempted moves, and each move has a $50 \%$ chance of being either translational or rotational.

\begin{tabular}{cccccc}
\hline$\eta$ & 0.2 & 0.3 & 0.4 & 0.5 & 0.6 \\
MC sweeps & $5 \times 10^{6}$ & $5 \times 10^{6}$ & $7.5 \times 10^{5}$ & $5 \times 10^{5}$ & $2 \times 10^{5}$ \\
\hline \hline
\end{tabular}

